\title{
Análise da Revisão Cochrane: Tratamentos Tópicos para a Psoríase do Couro-Cabeludo. Cochrane Database Syst Rev. 2016;2;CD009687.
}

\author{
Analysis of the Cochrane Review: Topical Treatments \\ for Scalp Psoriasis. Cochrane Database Syst Rev. \\ 2016;2:CD009687.
}

Catarina Soares QUEIRÓS', Gonçalo Silva DUARTEE ${ }^{2,3,4}$, João COSTA ${ }^{2,3,4}$, António VAZ-CARNEIRO $\rrbracket^{4,5}$

Acta Med Port 2017 Mar;30(3):163-168 - https://doi.org/10.20344/amp.8910

RESUMO

Independentemente do tipo de psoríase, até $79 \%$ dos doentes com esta patologia apresentam atingimento do couro cabeludo, sendo este frequentemente o primeiro local a mostrar sintomas da doença. Para além do prurido, as lesões avermelhadas e escamosas são visíveis e muitas vezes embaraçosas. A terapêutica tópica é geralmente a primeira opção para estes doentes, no entanto a ampla gama de tratamentos disponíveis pode muitas vezes dificultar a escolha e levar a um tratamento potencialmente desadequado. O objetivo desta revisão foi avaliar a eficácia e a segurança dos tratamentos tópicos para a psoríase do couro cabeludo. Foi efetuada uma revisão sistemática de acordo com a metodologia preconizada pela Cochrane, com vista à avaliação da redução da gravidade clínica da psoríase, melhoria da qualidade de vida e efeitos adversos que motivassem a suspensão do tratamento. Para tal, foram incluídos 59 estudos, com um total de 11561 participantes, tendo sido efetuadas 15 comparações entre os vários fármacos e veículos de aplicação testados. Os resultados obtidos sugerem que os corticosteroides de potência elevada ou muito elevada são mais eficazes que a vitamina $\mathrm{D}$. A combinação de um corticosteroide com a vitamina $\mathrm{D}$ tem apenas um benefício marginal em relação à monoterapia com corticosteroide, mas é superior à vitamina $\mathrm{D}$ isolada. Dado o perfil de segurança semelhante e o benefício apenas marginal da combinação corticosteroide com vitamina $\mathrm{D}$ sobre o corticosteroide isolado, a monoterapia com corticosteroide tópico parece ser aceitável para a terapia a curto prazo da psoríase do couro cabeludo.

Palavras-chave: Administração Tópica; Dermatoses do Couro Cabeludo/tratamento; Esteroides; Fármacos Dermatológicos/uso terapêutico; Psoriase/tratamento; Revisão Sistemática; Vitamina D

\section{ABSTRACT}

Regardless the psoriasis subtype, up to $79 \%$ of people with this skin condition present scalp involvement, which is often the first site to show symptoms of the disease. In addition to being itchy, the red and scaly lesions are usually easy to see, and may be embarrassing. Topical therapy is usually the first line of treatment; however the wide array of available interventions can make the choice difficult, and may even lead to an inadequate treatment. The objective of this review was to evaluate the efficacy and safety of topical treatments for scalp psoriasis. A systematic review was performed according to the methodology recommended by Cochrane in order to evaluate the clinical severity of psoriasis, quality of life, and adverse events that led to treatment discontinuation. To evaluate this, 59 studies were included, with a total of 11561 participants, and 15 comparisons were made between the various drugs and application vehicles tested. The principal conclusion is that corticosteroids of high or very high potency are more effective than vitamin D. The combination of a corticosteroid with vitamin D has only a marginal benefit over corticosteroid monotherapy, but is superior to vitamin D alone. Given the similar safety profile and only marginal benefit of the combination of corticosteroid with vitamin D over the corticosteroid alone, topical corticosteroid monotherapy appears to be fully acceptable for short-term therapy of scalp psoriasis.

Keywords: Administration, Topical; Dermatologic Agents/therapeutic use; Psoriasis/therapy; Scalp Dermatoses/therapy; Steroids; Systematic Review; Vitamin D

\section{INTRODUÇÃO}

A psoríase é uma doença inflamatória crónica da pele, que afeta aproximadamente $2 \%$ da população da Europa Ocidental e dos EUA., ${ }^{1,2}$ Existem diferentes tipos de psoríase, incluindo a pustular, guttata, inversa, eritrodérmica ou a psoríase crónica em placas (psoríase vulgar), esta última responsável por $90 \%$ dos casos. ${ }^{3}$ Independentemente do tipo de psoríase, até $79 \%$ dos doentes com esta patologia apresentam atingimento do couro cabeludo, sendo este frequentemente o primeiro local a mostrar sintomas da doença. Para além do prurido, as lesões avermelhadas e escamosas são visíveis e muitas vezes embaraçosas.

Perante um quadro de psoríase generalizada (que pode ou não atingir o couro cabeludo), o tratamento passa habitualmente pelo psoraleno combinado com ultravioleta $A$ (PUVA) ou por terapias sistémicas como o metotrexato, a ciclosporina ou agentes biológicos, entre outros. Por outro lado, na psoríase moderada do corpo e do couro cabeludo os tratamentos tópicos continuam a ser a primeira linha.

1. Serviço de Dermatologia. Hospital de Santa Maria. Lisboa. Portugal.

2. Laboratório de Farmacologia Clínica e Terapêutica. Faculdade de Medicina. Universidade de Lisboa. Lisboa. Portugal.

3. Clinical Pharmacology Unit. Instituto de Medicina Molecular. Lisboa. Portugal.

4. Centro de Estudos de Medicina Baseada na Evidência. Faculdade de Medicina. Universidade de Lisboa. Lisboa. Portugal.

5. Centro Colaborador Português da Rede Cochrane Iberoamericana. Lisboa. Portugal.

$\square$ Autor correspondente: António Vaz-Carneiro. avc@medicina.ulisboa.pt

Recebido: 01 de março de 2017 - Aceite: 01 de março de 2017 | Copyright @ Ordem dos Médicos 2017 
Neste sentido, existe uma ampla gama de opções terapêuticas, que incluem os corticosteróides, os análogos da vitamina $\mathrm{D}$, preparações à base de alcatrão, o ditranol, o ácido salicílico e o tacrolimus, entre outros.

A ampla gama de tratamentos disponíveis pode muitas vezes dificultar a escolha e levar a um tratamento potencialmente desadequado; esta situação, aliada ao facto de se tratar de uma patologia crónica, evidencia a importância de perceber quais as opções terapêuticas com melhor perfil benefício-risco.

\section{QUESTÃO CLÍNICA}

Quais são os tratamentos mais eficazes e mais seguros para a psoríase do couro-cabeludo?

\section{OBJECTIVOS}

Avaliar a eficácia e a segurança dos tratamentos tópicos para a psoríase do couro cabeludo.

\section{METODOLOGIA}

Esta revisão sistemática incluiu ensaios clínicos aleatorizados e controlados de grupos paralelos, cruzados (cross-over) ou com within-patient design. Foram incluídos participantes de todas as idades, com o diagnóstico clínico ou histológico de psoríase do couro-cabeludo. Foram considerados nas comparações as seguintes intervenções farmacológicas: corticosteroides (dipropionato de betametasona, propionato de clobetasol), viamina D (calcipotriol), combinações de corticosteroide com vitamina $\mathrm{D}$, combinações de corticosteroide com ácido salicílico, preparações à base de alcatrão, ciclopirox, tacrolimus, cocois e outras combinações contendo ditranol, óleo de coco, ureia e ácido salicílico.

Os ensaios clínicos incluídos foram identificados até ao dia 17 de agosto de 2015 através do recurso às seguintes bases de dados: Cochrane Skin Group Specialised Register; Cochrane Central Register of Controlled Trials;

Tabela 1 - Comparação entre corticosteroides e vitamina D na psoríase do couro cabeludo

\section{Doentes ou população: Pessoas com psoríase do couro cabeludo Intervenção: Corticosteroides Comparação: Vitamina D}

\begin{tabular}{|c|c|c|c|c|c|c|}
\hline \multirow{2}{*}{ Outcome } & \multicolumn{2}{|c|}{$\begin{array}{l}\text { Efeitos absolutos antecipados* } \\
\text { (IC 95\%) }\end{array}$} & \multirow{2}{*}{$\begin{array}{c}\text { Efeito } \\
\text { relativo } \\
\text { (IC 95\%) }\end{array}$} & \multirow{2}{*}{$\begin{array}{c}\text { Número de } \\
\text { participantes } \\
\text { (estudos) }\end{array}$} & \multirow{2}{*}{$\begin{array}{c}\text { Qualidade } \\
\text { da evidência } \\
\text { (GRADE) }\end{array}$} & \multirow{2}{*}{ Comentários } \\
\hline & $\begin{array}{l}\text { Risco com a } \\
\text { vitamina } D\end{array}$ & $\begin{array}{l}\text { Risco com o } \\
\text { corticosteroide }\end{array}$ & & & & \\
\hline $\begin{array}{l}\text { Número de } \\
\text { participantes com } \\
\text { resolução da lesão } \\
\text { (avaliada pelo } \\
\text { investigador) }\end{array}$ & 159 por 1000 & $\begin{array}{c}289 \text { por } 1000 \\
(241 \text { a } 346)\end{array}$ & $\begin{array}{c}\text { RR } 1,82 \\
(1,52 \text { a } 2,18)\end{array}$ & $\begin{array}{c}2180 \\
\text { (4 estudos) }\end{array}$ & Moderada $^{1}$ & \\
\hline $\begin{array}{l}\text { Número de } \\
\text { participantes que } \\
\text { obtiveram resposta } \\
\text { (avaliada pelo } \\
\text { investigador) }\end{array}$ & 251 por 1000 & $\begin{array}{c}525 \text { por } 1000 \\
(452 \text { a } 605)\end{array}$ & $\begin{array}{c}\text { RR } 2,09 \\
(1,80 \text { a } 2,41)\end{array}$ & $\begin{array}{c}1827 \\
\text { (3 estudos) }\end{array}$ & Alta & \\
\hline Qualidade de vida & 0 por 1000 & $\begin{array}{l}0 \text { por } 1000 \\
(0 \text { a } 0)\end{array}$ & - & (0 estudos) & & $\begin{array}{l}\text { Nenhum estudo } \\
\text { avaliou este outcome }\end{array}$ \\
\hline $\begin{array}{l}\text { Número de } \\
\text { participantes que } \\
\text { abandonou o } \\
\text { tratamento por efeitos } \\
\text { adversos }\end{array}$ & 53 por 1000 & $\begin{array}{l}12 \text { por } 1000 \\
(6 \text { a } 22)\end{array}$ & $\begin{array}{c}\text { RR } 0,22 \\
(0,11 \text { a } 0,42)\end{array}$ & $\begin{array}{c}2291 \\
\text { (4 estudos) }\end{array}$ & Moderada $^{2}$ & $\begin{array}{l}\text { Nenhum estudo } \\
\text { especificou o efeito } \\
\text { adverso responsável } \\
\text { pelo abandono do } \\
\text { tratamento }\end{array}$ \\
\hline $\begin{array}{l}\text { Número de } \\
\text { participantes que } \\
\text { obtiveram resposta } \\
\text { (avaliada pelo } \\
\text { participante) }\end{array}$ & 403 por 1000 & $\begin{array}{c}596 \text { por } 1000 \\
(516 \text { a } 693)\end{array}$ & $\begin{array}{c}\operatorname{RR} 1,48 \\
(1,28 \text { a } 1,72)\end{array}$ & $\begin{array}{c}1827 \\
\text { (3 estudos) }\end{array}$ & Moderada $^{3}$ & \\
\hline
\end{tabular}

IC: Intervalo de confiança

* O risco no grupo de intervenção (e o seu IC a 95\%) é baseado no risco assumido no grupo de comparação e no efeito relativo da intervenção (e no seu IC a 95\%). Sistema GRADE:

- Qualidade alta: os investigadores estão muito confiantes de que o efeito verdadeiro se situa próximo ao da estimativa do efeito.

- Qualidade moderada: os investigadores estão moderadamente confiantes na estimativa de efeito: o efeito verdadeiro provavelmente estará próximo da estimativa do efeito, mas há uma possibilidade de que seja substancialmente diferente.

- Qualidade baixa: a confiança dos investigadores na estimativa do efeito é limitada: o efeito verdadeiro pode ser substancialmente diferente do efeito estimado.

- Qualidade muito baixa: os investigadores têm muito pouca confiança na estimativa de efeito: o efeito verdadeiro é suscetível de ser substancialmente diferente do estimado. 1 Redução de um nível pelo risco de viés: três dos quatro estudos com obscure blinding na avaliação dos outcomes.; todos os estudos com ocultação de alocação pouco clara.

2 Redução de um nível pelo risco de viés: dois dos quatro estudos com obscure blinding obscuro na avaliação dos outcomes.; todos os estudos com ocultação de alocação pouco clara.

3 Redução de um nível pelo risco de viés: todos os estudos com ocultação de alocação pouco clara; um estudo com risco incerto de viés por informação seletiva; um estudo com obscure blinding na avaliação dos outcomes. 
MEDLINE; EMBASE; e LILACS. Em setembro de 2015 foram ainda incluídos ensaios clínicos registados nas seguintes plataformas: ISRCTN; US National Institutes of Health Ongoing Trials Register; Australian New Zealand Clinical Trials Registry; World Health Organization International Clinical Trials Registry Platform; e EU Clinical Trials Register. Os autores procederam ainda à identificação de ensaios clínicos adicionais através das referências bibliográficas dos ensaios já selecionados, bem como à pesquisa de resumos relevantes apresentados nas seguintes conferências nos últimos 12 anos: American Academy of Dermatology (AAD); European Academy of Dermatology and Venerology (EADV); Deutsche Dermatologische Gesellschaft (DDG); Psoriasis - From Gene to Clinic; Psoriasis International Network - Paris; e International Federation of Psoriasis Associations (IFPA) - Stockholm. Dois investigadores independentes selecionaram os estudos, extraíram os dados e avaliaram a qualidade metodológica dos ensaios. Os principais outcomes foram:

Outcomes primários:

- Redução da gravidade avaliada pelo clínico;

- Qualidade de vida:
- Eventos adversos responsáveis pela suspensão do tratamento, como reações alérgicas graves.

Outcomes secundários:

- Redução subjetiva da gravidade da psoríase;

- Eventos adversos minor que não motivassem a suspensão do tratamento (rash, prurido);

- Tempo livre de doença ou duração da resposta medida pela proporção de participantes que regressa aos scores basais durante o tratamento continuado ou após a sua descontinuação.

Os autores procederam à síntese de dados e exprimiram os resultados dos estudos individuais sob a forma de risco relativo (RR) com intervalos de confiança (IC) a 95\% para outcomes dicotómicos, e diferenças médias e IC a $95 \%$ para outcomes contínuos. Foram estudadas com especial ênfase as 3 comparações com maior relevância clínica: corticosteroide versus vitamina $\mathrm{D}$, combinação de corticosteroide e vitamina $D$ vs corticosteroide em monoterapia, e combinação de corticosteroide e vitamina $D$ versus vitamina $\mathrm{D}$ em monoterapia; a qualidade geral da evidência para estas três comparações foi moderada.

Tabela 2 - Comparação entre a terapêutica combinada (corticosteroide e vitamina D) e os corticosteroides na psoríase do couro cabeludo

Doentes ou população: Pessoas com psoríase do couro cabeludo

Intervenção: Combinação de corticosteroide e vitamina D

Comparação: Corticosteroide

\begin{tabular}{|c|c|c|c|c|c|c|}
\hline \multirow{2}{*}{ Outcome } & \multicolumn{2}{|c|}{$\begin{array}{l}\text { Efeitos absolutos antecipados* } \\
\text { (IC 95\%) }\end{array}$} & \multirow{2}{*}{$\begin{array}{l}\text { Efeito } \\
\text { relativo } \\
\text { (IC 95\%) }\end{array}$} & \multirow{2}{*}{$\begin{array}{l}\text { Número de } \\
\text { participantes } \\
\text { (estudos) }\end{array}$} & \multirow{2}{*}{$\begin{array}{c}\text { Qualidade } \\
\text { da evidência } \\
\text { (GRADE) }\end{array}$} & \multirow{2}{*}{ Comentários } \\
\hline & $\begin{array}{l}\text { Risco com o } \\
\text { corticosteroide }\end{array}$ & $\begin{array}{l}\text { Risco com a } \\
\text { combinação }\end{array}$ & & & & \\
\hline $\begin{array}{l}\text { Número de participantes } \\
\text { com resolução da } \\
\text { lesão (avaliada pelo } \\
\text { investigador) }\end{array}$ & 287 por 1000 & $\begin{array}{c}350 \text { por } 1000 \\
(310 \text { a } 391)\end{array}$ & $\begin{array}{c}\text { RR } 1,22 \\
(1,08 \text { a } 1,36)\end{array}$ & $\begin{array}{c}2474 \\
\text { (4 estudos) }\end{array}$ & Moderada $^{1}$ & \\
\hline $\begin{array}{l}\text { Número de participantes } \\
\text { que obtiveram } \\
\text { resposta (avaliada pelo } \\
\text { investigador) }\end{array}$ & 546 por 1000 & $\begin{array}{l}628 \text { por } 1000 \\
(579 \text { a } 683)\end{array}$ & $\begin{array}{c}\text { RR } 1,15 \\
(1,06 \text { a } 1,25)\end{array}$ & $\begin{array}{c}2444 \\
\text { (3 estudos) }\end{array}$ & Moderada $^{2}$ & \\
\hline Qualidade de vida & 0 por 1000 & $\begin{array}{l}0 \text { por } 1000 \\
(0 \text { a } 0)\end{array}$ & - & (0 estudos) & & $\begin{array}{l}\text { Nenhum estudo } \\
\text { avaliou este outcome }\end{array}$ \\
\hline $\begin{array}{l}\text { Número de participantes } \\
\text { que abandonou o } \\
\text { tratamento por efeitos } \\
\text { adversos }\end{array}$ & 12 por 1000 & $\begin{array}{c}11 \text { por } 1000 \\
(5 \text { a } 23)\end{array}$ & $\begin{array}{c}\text { RR } 0,88 \\
(0,42 \text { a } 1,88)\end{array}$ & $\begin{array}{c}2433 \\
\text { (3 estudos) }\end{array}$ & Moderada $^{3}$ & $\begin{array}{l}\text { Nenhum estudo } \\
\text { especificou o efeito } \\
\text { adverso responsável } \\
\text { pelo abandono do } \\
\text { tratamento }\end{array}$ \\
\hline $\begin{array}{l}\text { Número de participantes } \\
\text { que obtiveram } \\
\text { resposta (avaliada pelo } \\
\text { participante) }\end{array}$ & 613 por 1000 & $\begin{array}{c}692 \text { por } 1000 \\
(649 \text { a } 735)\end{array}$ & $\begin{array}{c}\text { RR } 1,13 \\
(1,06 \text { a } 1,20)\end{array}$ & $\begin{array}{c}2226 \\
\text { (2 estudos) }\end{array}$ & Alta & \\
\hline
\end{tabular}

IC: Intervalo de confiança

* O risco no grupo de intervenção (e o seu IC a 95\%) é baseado no risco assumido no grupo de comparação e no efeito relativo da intervenção (e no seu IC a 95\%).

Sistema GRADE:

- Qualidade alta: os investigadores estão muito confiantes de que o efeito verdadeiro se situa próximo ao da estimativa do efeito.

Qualidade moderada: os investigadores estão moderadamente confiantes na estimativa de efeito: o efeito verdadeiro provavelmente estará próximo da estimativa do efeito, mas

há uma possibilidade de que seja substancialmente diferente.

- Qualidade baixa: a confiança dos investigadores na estimativa do efeito é limitada: o efeito verdadeiro pode ser substancialmente diferente do efeito estimado.

- Qualidade muito baixa: os investigadores têm muito pouca confiança na estimativa de efeito: o efeito verdadeiro é suscetível de ser substancialmente diferente do estimado.

1 Redução de um nível porque o IC cruza a linha do limiar de diferença mínima importante (DMI): diferença estatisticamente significativa de importância clínica incerta.

2 Redução de um nível por inconsistência devido a heterogeneidade moderada $\left(I^{2}=35 \%\right)$.

3 Redução de um nível por imprecisão uma vez que o IC cruza a linha do limiar de DMI: incerteza quanto à existência de alguma diferença. 


\section{RESULTADOS}

Foram incluídos 59 estudos, com um total de 11561 participantes, tendo sido efetuadas 15 comparações entre os vários fármacos testados. A eficácia das intervenções farmacológicas foi avaliada pelos investigadores e/ou pelos participantes, através do número de participantes que respondeu ou resolveu totalmente as lesões.

Nas comparações de fármacos individuais com o seu veículo (sem o princípio ativo), os corticosteroides, a vitamina $D$ ou a combinação destes mostraram eficácia superior ao veículo respetivo; à exceção de alguns ensaios com corticosteroides, não existem dados fiáveis que permitam determinar o benefício adicional de veículos específicos sobre a eficácia e segurança de um dado princípio ativo.
Nas comparações diretas entre dois fármacos, os corticosteroides foram superiores à vitamina $D$ (Tabela 1). A combinação de um corticosteroide com vitamina $D$ foi mais eficaz que o corticoide em monoterapia, no entanto clinicamente este benefício pode não ser relevante (Tabela 2). Esta combinação (corticosteroide e vitamina $D$ ) foi também superior à vitamina $D$ isolada (Tabela 3). Comparados entre si, os corticosteroides tenderam a ser igualmente eficazes e com um risco de eventos adversos semelhantes, independentemente da sua potência. Para além disso, parece não haver diferença entre a aplicação dos corticosteroides uma ou duas vezes por dia. Não foi possível avaliar suficientemente a eficácia e a segurança de outros tratamentos tópicos, tais como o ácido salićlico, o alcatrão ou o ditranol.

Tabela 3 - Comparação entre a terapêutica combinada (corticosteroide e vitamina D) e a vitamina D na psoríase do couro cabeludo

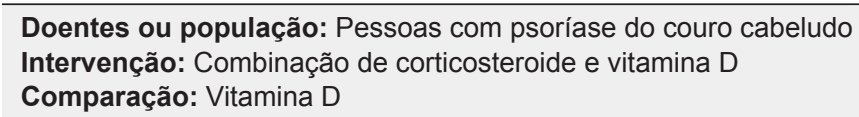

\begin{tabular}{|c|c|c|c|c|c|c|}
\hline \multirow{2}{*}{ Outcome } & \multicolumn{2}{|c|}{$\begin{array}{l}\text { Efeitos absolutos antecipados* } \\
\text { (IC 95\%) }\end{array}$} & \multirow{2}{*}{$\begin{array}{l}\text { Efeito } \\
\text { relativo } \\
\text { (IC 95\%) }\end{array}$} & \multirow{2}{*}{$\begin{array}{l}\text { Número de } \\
\text { participantes } \\
\text { (estudos) }\end{array}$} & \multirow{2}{*}{$\begin{array}{c}\text { Qualidade } \\
\text { da evidência } \\
\text { (GRADE) }\end{array}$} & \multirow{2}{*}{ Comentários } \\
\hline & $\begin{array}{l}\text { Risco com a } \\
\text { vitamina D }\end{array}$ & $\begin{array}{l}\text { Risco com a } \\
\text { combinação }\end{array}$ & & & & \\
\hline $\begin{array}{l}\text { Número de participantes } \\
\text { com resolução da } \\
\text { lesão (avaliada pelo }\end{array}$ & 145 por 1000 & $\begin{array}{c}330 \text { por } 1000 \\
(270 \text { a } 402)\end{array}$ & $\begin{array}{c}\text { RR } 2,28 \\
(1,87 \text { a } 2,78)\end{array}$ & $\begin{array}{c}2008 \\
\text { (4 estudos) }\end{array}$ & Alta & \\
\hline
\end{tabular}

lesão (avaliada pelo investigador)

\begin{abstract}
Número de participantes que obtiveram resposta (avaliada pelo investigador)
\end{abstract}

Qualidade de vida

Número de participantes que abandonou o tratamento por efeitos adversos

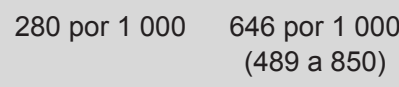

0 por 1000

0 por 1000

(0 a 0$)$
56 por 1000
11 por 1000 (6 a 20)

RR 0,19 $(0,33$ a 1,36$)$

RR 2,31

$(1,75$ a 3,04$)$
2222
(3 estudos)

Moderada $^{1}$

\section{Número de participantes que obtiveram resposta (avaliada pelo participante)}

427 por $1000 \quad \begin{array}{r}751 \text { por } 1000 \\ (623 \text { a } 905)\end{array}$

RR 1,76
$(1,46$ a 2,12$)$

(0 estudos)

1970

(3 estudos)

Alta

Nenhum estudo especificou o efeito adverso responsável pelo abandono do tratamento

IC: Intervalo de confiança

* O risco no grupo de intervenção (e o seu IC a 95\%) é baseado no risco assumido no grupo de comparação e no efeito relativo da intervenção (e no seu IC a 95\%). Sistema GRADE:

- Qualidade alta: os investigadores estão muito confiantes de que o efeito verdadeiro se situa próximo ao da estimativa do efeito.

- Qualidade moderada: os investigadores estão moderadamente confiantes na estimativa de efeito: o efeito verdadeiro provavelmente estará próximo da estimativa do efeito, mas há uma possibilidade de que seja substancialmente diferente.

- Qualidade baixa: a confiança dos investigadores na estimativa do efeito é limitada: o efeito verdadeiro pode ser substancialmente diferente do efeito estimado.

- Qualidade muito baixa: os investigadores têm muito pouca confiança na estimativa de efeito: o efeito verdadeiro é suscetível de ser substancialmente diferente do estimado.

1 Redução de um nível por inconsistência devido a heterogeneidade moderada $\left(I^{2}=81 \%\right)$, que pode ser devida à frequência de aplicação diferente nos estudos (uma vez versus duas vezes ao dia).

2 Redução de um nível por inconsistência devido a heterogeneidade moderada $\left(I^{2}=77 \%\right.$ ), que pode ser devida à frequência de aplicação diferente nos estudos (uma vez versus duas vezes ao dia). 
Os eventos adversos mais comuns dos tratamentos tópicos foram a irritação, o prurido e a dor no local de aplicação. Eventos adversos noutros locais do corpo foram raros e provavelmente não causados pelas intervenções. O risco de abandono terapêutico devido a eventos adversos foi maior no grupo alocado à vitamina $D$ do que os que aplicaram um corticosteroide tópico ou a combinação de ambos, não havendo diferenças no risco de eventos adversos entre estes últimos. Nenhum estudo relatou o tipo de evento adverso que motivou a suspensão do tratamento.

Devido à fraca informação nesse âmbito, não foi possível avaliar qual o tratamento que mais influenciou a qualidade de vida. Um dos estudos demonstrou uma melhoria mais acentuada da qualidade de vida nos participantes submetidos à combinação de corticosteroide com vitamina D comparativamente aos tratados com vitamina $D$ em monoterapia, no entanto a maioria dos estudos simplesmente não mediu o impacto na qualidade de vida. Nenhum dos estudos identificados forneceu dados sobre o tempo até a recaída, conforme definido no protocolo para esta revisão; por essa razão, este outcome não foi avaliado.

As limitações no desenho e implementação dos estudos variaram consideravelmente entre os estudos incluídos, sendo que apenas onze estudos explicaram claramente o método de aleatorização e apenas quatro relataram como foi ocultada a alocação, o que representa um risco potencial de viés de seleção. Para além disso, cerca de um terço dos ensaios foi classificado como de risco elevado ou desconhecido quanto a perdas de seguimento.

\section{CONCLUSÃO}

Os resultados desta revisão sistemática sugerem que os corticosteroides de potência elevada ou muito elevada são mais eficazes que a vitamina $D$. A combinação de um corticosteroide com a vitamina $D$ tem apenas um benefício marginal em relação à monoterapia com corticosteroide, mas é superior à vitamina $D$ isolada. Não parece haver diferenças quanto à eficácia entre os corticosteroides de potência moderada, alta e muito alta. Não há evidência suficiente para permitir uma conclusão final sobre se o ácido salicílico traz ou não benefício adicional quando usado em combinação com corticosteroides.

Para o tratamento a curto prazo, a combinação de corticosteroide com vitamina D e a monoterapia com corticosteroide não diferem quanto ao risco de reações adversas, sendo que ambas foram melhor toleradas que a vitamina $D$ isolada. Na terapia a longo prazo, a combinação dos dois compostos causou menos eventos adversos e abandonos secundários aos mesmos do que a vitamina $\mathrm{D}$ em monoterapia.

Na opinião dos autores, dado o perfil de segurança se- melhante e o benefício apenas marginal da combinação corticosteroide com vitamina $\mathrm{D}$ sobre o corticosteroide isolado, a monoterapia com corticosteroide tópico parece ser uma opção aceitável para a terapia a curto prazo da psoríase do couro cabeludo.

\section{COMENTÁRIO}

Esta revisão pretendeu incluir todos os tratamentos tópicos disponíveis e deparou-se com uma grande variedade de intervenções diferentes. Os estudos incluídos avaliaram populações representativas e utilizaram comparações com os veículos e com outros princípios ativos, o que permitiu um julgamento claro sobre a eficácia das intervenções avaliadas. Foram identificados ensaios multicêntricos que incluíram grandes populações de estudo para as intervenções mais estabelecidas, tais como os corticosteroides tópicos, a vitamina $D$ e a combinação de ambos. A evidência da eficácia e segurança destas terapêuticas está de acordo com as atuais recomendações de consenso europeu, americano e asiático. ${ }^{4,5,6}$ Para intervenções menos estabelecidas, como é o caso do alcatrão ou do ácido salicílico em combinação com os corticosteroides, a avaliação da consistência dos resultados entre os estudos foi limitada ou não viável, o que pode comprometer a validade externa.

A avaliação global de quase todos os tratamentos incluídos foi limitada pelas curtas durações dos estudos (maioria com seis meses de duração). A avaliação de preparações de alcatrão e de outros produtos como o ciclopirox olamina, o tacrolimus, a combinação do ditranol com ureia ou a combinação de corticosteróides com ácido salicílico foi limitada devido à falta de evidência. O couro cabeludo é uma parte visível do corpo e difícil de tratar devido à presença de cabelo, desta forma, os ensaios futuros devem avaliar a tolerância dos pacientes às preparações tópicas, o que pode envolver a avaliação de cheiro, viscosidade ou oleosidade, entre outros. Esta é uma questão importante com grande influência na qualidade de vida e na adesão do paciente.

\section{IMPLICAÇÕES PARA A PRÁTICA CLÍNICA}

- Os corticosteroides em monoterapia e a combinação de um corticosteroide com a vitamina $D$ são as alternativas que aliam uma maior eficácia a um menor risco de efeitos adversos.

- Dado o perfil de segurança semelhante e benefício acrescido apenas marginal da combinação corticosteroide com vitamina $\mathrm{D}$ face à monoterapia com corticosteroide, os corticosteroides tópicos são uma opção terapêutica a curto prazo da psoríase do couro cabeludo.

- Não parecem existir diferenças entre a eficácia dos corticosteroides de potência moderada, alta e muito alta.

\section{REFERÊNCIAS}

1. Schlager J, Rosumeck S, Werner R, Jacobs A, Schmitt J, Schlager C, Nast A. Topical treatments for scalp psoriasis. Cochrane Database Syst Rev. 2016;2:CD009687.

2. Van de Kerkhof PC, Franssen ME. Psoriasis of the scalp. Diagnosis and management. Am J Clin Dermatol. 2001;2:159-65.

3. Griffiths CE, Barker JN. Pathogenesis and clinical features of psoriasis. 
Lancet. 2007:370:263-71.

4. Chan CS, Van Voorhees AS, Lebwohl MG, Korman NJ, Young M, Bebo BF Jr, et al. Treatment of severe scalp psoriasis: from the Medical Board of the National Psoriasis Foundation. J Am Acad Dermatol. 2009;60:962-71.

5. Frez ML, Asawanonda P, Gunasekara C, Koh C, Loo S, Oon HH, et al.
Recommendations for a patient-centred approach to the assessment and treatment of scalp psoriasis: a consensus statement from the Asia Scalp Psoriasis Study Group. J Dermatolog Treat. 2014;1:38-45.

6. Ortonne J, Chimenti S, Luger T, Puig L, Reid F, Trueb RM. Scalp psoriasis: European consensus on grading and treatment algorithm. J Eur Acad Dermatol Venereol. 2009;23:1435-44.

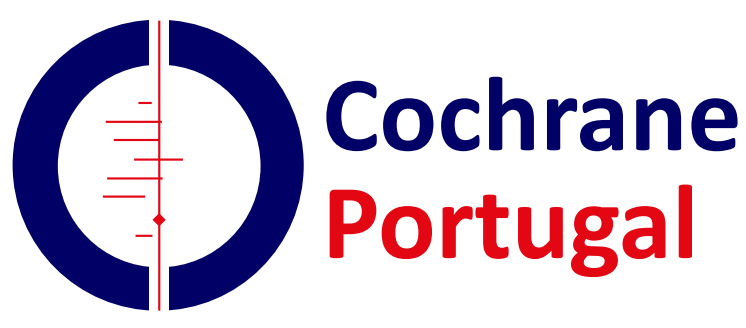

